Case

Report

\title{
Indocyanine Green Fluorescence Navigation Thoracoscopic Metastasectomy for Pulmonary Metastasis of Hepatocellular Carcinoma
}

\author{
Naoya Kawakita, PhD, Hiromitsu Takizawa, MD, PhD, Kazuya Kondo, MD, PhD, \\ Shoji Sakiyama, MD, PhD, and Akira Tangoku, MD, PhD
}

\begin{abstract}
Indocyanine green can selectively accumulate in primary hepatocellular carcinoma (HCC) and extrahepatic metastases. We report a patient who underwent resection of pulmonary metastasis of HCC using a thoracoscopic near-infrared imaging system and fluorescent navigation surgery. A 66-year-old man with suspicion of pulmonary metastasis of HCC was referred to our hospital. Indocyanine green was injected intravenously at a dose of $0.5 \mathrm{mg} / \mathrm{kg}$ body weight, $20 \mathrm{~h}$ before thoracoscopic surgery. An endoscopic indocyanine green nearinfrared fluorescence imaging system showed clear blue fluorescence, indicating pulmonary metastasis of HCC in a lingular segment. We performed wide wedge resection using the fluorescence image for navigation to confirm the surgical margins. The specimen was histologically confirmed as a pulmonary metastasis of HCC. In conclusion, thoracoscopic indocyanine green near-infrared fluorescence imaging for pulmonary metastases of $\mathrm{HCC}$ is useful in identifying tumor locations and ensuring resection margins.
\end{abstract}

Keywords: indocyanine green, hepatocellular carcinoma, near-infrared imaging

\section{Introduction}

Indocyanine green (ICG) can selectively accumulate in primary hepatocellular carcinoma (HCC) and extrahepatic metastases. ${ }^{1-3)}$ Recent reports demonstrated that ICG fluorescence imaging for HCC patients presents clear boundaries between tumors and normal tissue. This is the first report of thoracoscopic metastasectomy using ICG fluorescence for a patient with pulmonary metastasis of HCC.

Department of Thoracic, Endocrine Surgery and Oncology, Institute of Health Bioscience, The University of Tokushima Graduate School, Tokushima, Tokushima, Japan

Received: December 20, 2015; Accepted: February 29, 2016 Corresponding author: Naoya Kawakita, PhD. Department of Thoracic and Endocrine Surgery and Oncology, Institute of Health Biosciences, The University of Tokushima Graduate School, 3-18-15, Kuramoto- cho, Tokushima, Tokushima 770-8503, Japan Email: kawakita.naoya@clin.med.tokushima-u.ac.jp (C)2016 The Editorial Committee of Annals of Thoracic and Cardiovascular Surgery. All rights reserved.

\section{Case Report}

A 66-year-old man was referred to our thoracic surgery department for resection of HCC pulmonary metastasis. He had a history of chronic hepatitis B and had undergone right hepatic lobectomy for HCC 8 years prior. Seven years prior, follow-up computed tomography (CT) showed pulmonary metastatic lesions: an 8-mm nodule in the right upper lobe, an 8-mm nodule in the left upper lobe, and a 38-mm Bottalo's lymph node metastasis. Although the patient underwent three regimens of chemotherapy, these metastases progressed. Therefore, oral administration of sorafenib was started 6 years prior to the present admission. Chest CT demonstrated that the size of these lesions had decreased, and the pulmonary metastasis in the right upper lobe and the Bottalo's lymph node metastasis disappeared 1 year after the induction of sorafenib, with persistence of response. However, only the metastasis in the left upper lobe showed regrowth (Fig. 1A), and resection of the metastasis was scheduled. ICG was injected intravenously at a dose of $0.5 \mathrm{mg} / \mathrm{kg}$ 


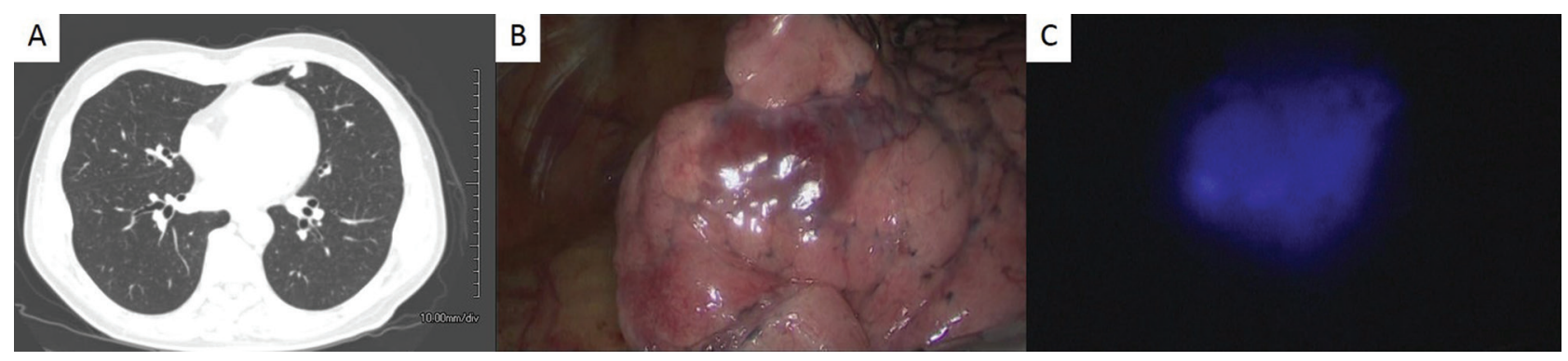

Fig. 1 (A) A chest computed tomography image shows a 15-mm nodule in the left upper lobe. (B) White light mode shows a reddish-brown change at the pleural surface of the lingular segment. (C) ICG fluorescent mode shows clear blue fluorescence in the lingular segment. ICG: indocyanine green

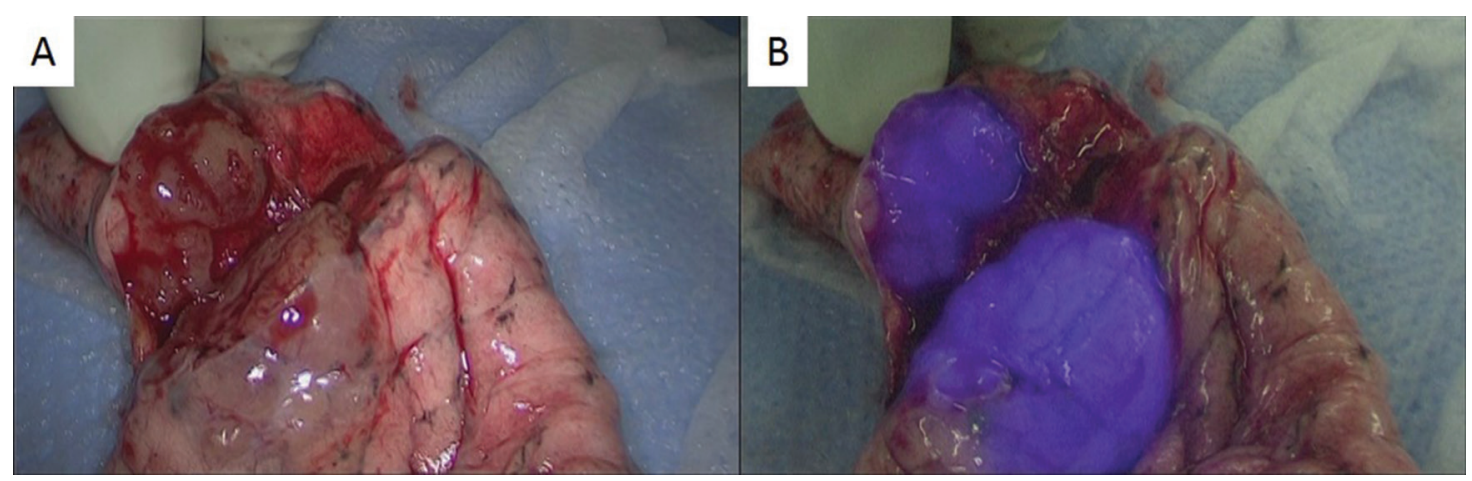

Fig. 2 (A) A section of the resected specimen. (B) ICG fluorescent mode shows clear blue fluorescence at the tumor. ICG: indocyanine green

body weight, $20 \mathrm{~h}$ before the operation. A 3-cm incision in the 4th intercostal space (ICS) along the anterior axillary line, a 3-cm incision in the 6th ICS along the posterior axillary line, and a 2-cm incision in the 8th ICS along the middle axillary line were made. The thoracic cavity was explored with an endoscopic ICG near-infrared fluorescence (NIF) imaging system (D-LIGHT P, Karl Storz, Germany). White light mode showed a reddish-brown change at the pleural surface of the lingular segment (Fig. 1B), and a whitish pleural thickening around the aortic arch. ICG fluorescent mode showed clear blue fluorescence in the lingular segment (Fig. 1C), while no ICG fluorescence was observed at Bottalo's lymph node. The center of the tumor was grasped with lung grasping forceps under the guidance of ICG-NIF imaging, and wide wedge resection was performed with an automatic suturing instrument. Although we had planned resection of Bottalo's lymph node to evaluate the viability of HCC, we aborted this plan due to the sclerosis and firm adhesion of the lymph node. A section of the resected specimen also showed ICG fluorescence (Fig. 2). Histologically, the tumor was confirmed as pulmonary metastasis of HCC, and the surgical margins were negative. A CT scan showed that the size of Bottalo's lymph node remained unchanged without any treatment for 6 months after the operation.

\section{Discussion}

Intrahepatic recurrence after hepatectomy for HCC is common. However, extrahepatic metastases occur in $37 \%$ of patients with HCC recurrence, and the most frequent site of the first detectable metastasis is the lung. ${ }^{4)}$ Pulmonary metastasectomy for pulmonary metastasis of HCC has been reported to play an important role in selected patients, particularly in those with a limited number of pulmonary metastases, unilateral metastases, or requiring single pulmonary surgery. ${ }^{5-7)}$

Sorafenib is the first drug that has been shown to be effective for patients with advanced hepatocellular carcinoma. ${ }^{8)}$ Our patient was administered sorafenib due to the presence of pulmonary metastases and a mediastinal lymph node metastasis. One year after the administration of sorafenib, we scheduled resection of a pulmonary metastasis that showed sorafenib chemoresistance, although the other pulmonary metastasis and mediastinal lymph node metastasis had disappeared. 
ICG emits light with a peak wavelength of approximately $830 \mathrm{~nm}$ when illuminated with NIF, and the ICG-NIF system is widely used in cancer diagnosis and surgery. ${ }^{9}$ Ishizawa et al. demonstrated selective accumulation of ICG to HCC using the ICG-NIF system. ${ }^{2)}$ Satou et al. reported that ICG fluorescence could also be observed at extrahepatic metastases. ${ }^{3)}$ They reported that they had succeeded in identifying one pulmonary metastases out of two lesions that were explored using an ICG-NIF system; however, they reported that the excitation light was unable to reach another three lesions through a thoracotomy because their system was not an endoscopic device. In the present study, we used an endoscopic ICG-NIF system, which is able to access the entire thoracic cavity, and clear blue fluorescence was observed at the metastatic site. ICG fluorescence is detectable when a target lesion exists within $1 \mathrm{~cm}$ from the organ surface, ${ }^{10,11)}$ and small metastatic lesions of $\mathrm{HCC}$ with a diameter of $3 \mathrm{~mm}$ were reported detectable by an ICG-NIF system when the lesions were close to the pleural surface. ${ }^{1,3)}$ Therefore, the ICG-NIF system may be useful in identifying small metastatic lesions without the need of preoperative marking and exploration of small lesions unidentified by preoperative imaging.

Use of thoracoscopic surgery is becoming more widespread due to its reduced invasiveness. ${ }^{12)}$ Indeed, thoracoscopic surgery was performed for approximately $80 \%$ of pulmonary metastasectomies in Japan. ${ }^{13)}$ Patients with pulmonary metastasis of HCC are often not good candidates for invasive surgery due to poor liver function caused by cirrhosis or hepatectomy; therefore, thoracoscopic surgery is useful for such patients.

One drawback of thoracoscopic surgery is the difficulty in confirming the resection margins, due to the inability to palpate the tumor. In our case, the tumor location could be visualized by the ICG-NIF system, allowing performance of wide wedge resection with sufficient distance from the fluorescent area; a negative surgical margin during thoracoscopic metastasectomy is considered to be a good prognostic factor. ${ }^{14,15)}$ If the tumor is located deep in the visceral pleura that it is undetectable using the ICG-NIF system, preoperative tumor marking or thoracotomy for palpation is necessary. Segmentectomy or lobectomy must also be considered for such patients with deeply located metastases to ensure sufficient surgical margins.

\section{Conclusion}

Thoracoscopic ICG-NIF for pulmonary metastases of $\mathrm{HCC}$ is useful in identifying tumor locations and ensuring resection margins.

\section{Disclosure Statement}

The authors declare that they have no competing interests.

\section{References}

1) Gotoh K, Yamada T, Ishikawa O, et al. A novel imageguided surgery of hepatocellular carcinoma by indocyanine green fluorescence imaging navigation. J Surg Oncol 2009; 100: 75-9.

2) Ishizawa $T$, Fukushima $N$, Shibahara J, et al. Real-time identification of liver cancers by using indocyanine green fluorescent imaging. Cancer 2009; 115: 2491-504.

3) Satou S, Ishizawa $T$, Masuda $K$, et al. Indocyanine green fluorescent imaging for detecting extrahepatic metastasis of hepatocellular carcinoma. J Gastroenterol 2013; 48: 1136-43.

4) Katyal S, Oliver JH, Peterson MS, et al. Extrahepatic metastases of hepatocellular carcinoma. Radiology 2000; 216: 698-703.

5) Kuo SW, Chang YL, Huang PM, et al. Prognostic factors for pulmonary metastasectomy in hepatocellular carcinoma. Ann Surg Oncol 2007; 14: 992-7.

6) Chen F, Sato K, Fujinaga T, et al. Pulmonary resection for metastases from hepatocellular carcinoma. World J Surg 2008; 32: 2213-7.

7) Nakajima J, Tanaka M, Matsumoto J, et al. Appraisal of surgical treatment for pulmonary metastasis from hepatocellular carcinoma. World J Surg 2005; 29: 715-8.

8) Llovet JM, Ricci S, Mazzaferro V, et al. Sorafenib in advanced hepatocellular carcinoma. N Engl J Med 2008; 359: 378-90.

9) Zelken JA, Tufaro AP. Current trends and emerging future of indocyanine green usage in surgery and oncology: an update. Ann Surg Oncol 2015; 22(Suppl 3): 1271-83.

10) Kim S, Lim YT, Soltesz EG, et al. Near-infrared fluorescent type II quantum dots for sentinel lymph node mapping. Nat Biotechnol 2004; 22: 93-7.

11) Kitai $T$, Inomoto $T$, Miwa $M$, et al. Fluorescence navigation with indocyanine green for detecting sentinel lymph nodes in breast cancer. Breast Cancer 2005; 12: 211-5.

12) Yim AP, Wan S, Lee TW, et al. VATS lobectomy reduces cytokine responses compared with conventional surgery. Ann Thorac Surg 2000; 70: 243-7.

13) Masuda M, Kuwano H, Okumura M, et al. Thoracic and cardiovascular surgery in Japan during 2012: annual report by The Japanese Association for Thoracic Surgery. Gen Thorac Cardiovasc Surg 2014; 62: 734-64.

14) Chua TC, Scolyer RA, Kennedy CW, et al. Surgical management of melanoma lung metastasis: an analysis of survival outcomes in 292 consecutive patients. Ann Surg Oncol 2012; 19: 1774-81.

15) Kim S, Ott HC, Wright CD, et al. Pulmonary resection of metastatic sarcoma: prognostic factors associated with improved outcomes. Ann Thorac Surg 2011; 92: 1780-6; discussion 1786-7. 